CONF-960493--3

UCRL-JC-123203

PREPRINT

\title{
Low-Cost Automated Fiber Pigtailing Machine
}

\section{$A P 12193$ \\ OSTI}

\author{
Oliver T. Strand
}

This paper was prepared for submittal to the Optical Society of America, Topical Meeting on Integrated Photonics Research Boston, MA

April 29-May 3, 1996

\section{January, 1996}

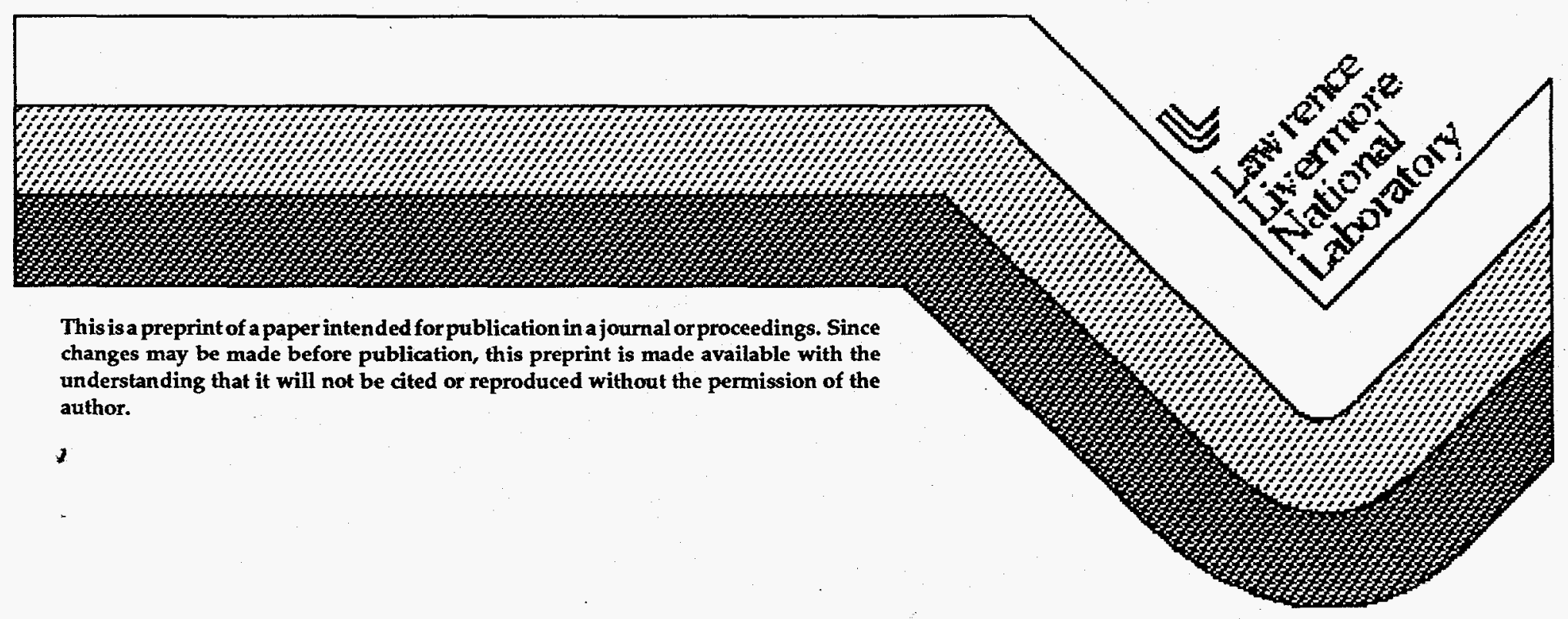




\section{DISCLAIMER}

This document was prepared as an account of work sponsored by an agency of the United States Government. Neither the United States Government nor the University of California nor any of their employees, makes any warranty, express or implied, or assumes any legal liability or responsibility for the accuracy, completeness, or usefulness of any information, apparatus, product, or process disclosed, or represents that its use would not infiringe privately owned rights. Reference herein to any specific commercial products, process, or service by trade name, trademark, manufacturer, or otherwise, does not necessarily constitute or imply its endorsement, recommendation, or favoring by the United States Govermment or the University of California. The views and opinions of authors expressed herein do not necessarily state or reflect those of the United States Government or the University of California, and shall not be used for advertising or product endorsement purposes. 


\section{DISCLAMMER}

Portions of this document may be illegible in electronic image products. Images are produced from the best available original document. 


\title{
LOW-COST AUTOMATED FIBER PIGTAILING MACHINE
}

\author{
Oliver T. Strand \\ Lawrence Livermore National Laboratory \\ Livermore, CA
}

Introduction

At present, the high cost of optoelectronic (OE) devices is caused in part by the laborintensive processes involved with packaging. Automating the packaging processes should result in a significant cost reduction. One of the most labor-intensive steps is aligning and attaching the fiber to the OE device, the so-called pigtailing process. The goal of this 2-year ARPA-funded project is to design and build 3 low-cost machines to perform sub-micron alignments and attachments of single-mode fibers to different OE devices. These Automated Fiber Pigtailing Machines (AFPMs) are intended to be compatible with a manufacturing environment and have a modular design for standardization of parts and machine vision for maximum flexibility. This work is a collaboration among Uniphase Telecommunications Products (formerly United Technologies Photonics, UTP), Ortel, Newport/Klinger, the Massachusetts Institute of Technology Manufacturing Institute (MIT), and Lawrence Livermore National Laboratory (LLNL). UTP and Ortel are the industrial partners for whom two of the AFPMs are being built. MIT and LLNL make up the design and assembly team of the project, while Newport/Klinger is a potential manufacturer of the AFPM and provides guidance to ensure that the design of the AFPM is marketable and compatible with a manufacturing environment. The AFPM for UTP will pigtail $\mathrm{LiNbO}_{3}$ waveguide devices and the AFPM for Ortel will pigtail photodiodes. Both of these machines will contain proprietary information, so the third AFPM, to reside at LLNL, will pigtail a non-proprietary waveguide device for demonstrations to US industry.

\section{AFPM Design}

The AFPM (Figure 1) is designed to be lowcost $(<\$ 150 \mathrm{~K})$, modular, flexible, and compatible with a manufacturing environment. The performance goals of the AFPM are to perform each pigtailing operation in less than 3 minutes (including the epoxy curing time) and to operate unattended for up to 1 hour. During the alignment operation, the $\mathrm{OE}$ device is held fixed while the ends of the fibers are moved by the high-precision stages. These machines incorporate a 2-step procedure to perform submicron alignments. The first step uses computer vision to align the fiber sufficiently close (few microns) to the $\mathrm{OE}$ device to couple light between the fiber and the device; the second step then achieves the sub-micron alignment by maximizing the light throughput. The overall design of the AFPM was carefully considered to minimize the requirements for high-precision machining tolerances; this greatly reduced the cost of building the AFPM. The modular nature of the AFPM means that each machine may be easily customized for a particular application.

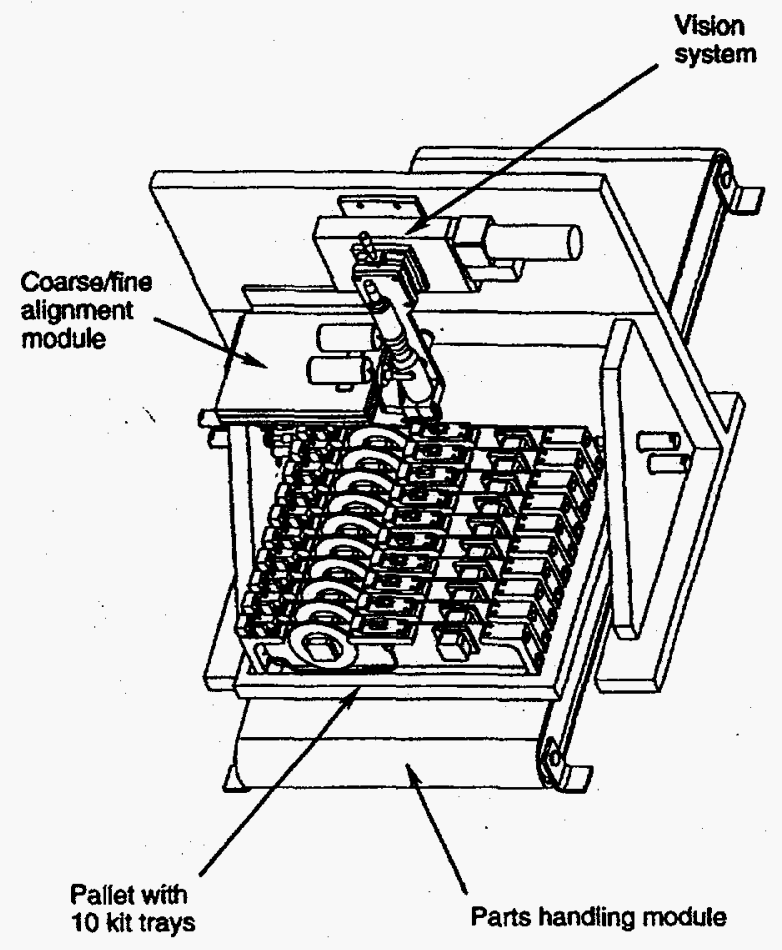

Figure 1. The Ortel AFPM has one high-precision stage mounted on the left side of the frame. 
For this project, a basic set of modules was designed to build prototype AFPMs which can pigtail devices with 3 very different geometries including a photodiode and two different types of waveguide devices; a different set of the same modules would allow laser diodes to be pigtailed, for example. Considerable effort was put into the parts handling scheme which allows the AFPM to operate unattended for significant amounts of time. A conveyor system is provided to deliver the $O E$ devices to the alignment stages and vision system. A description of several modules is given in the following sub-sections.

\section{Vision Module}

The vision system greatly reduces mechanical fixturing constraints by requiring the critical components to be positioned only within the field of view of the camera. The field of view for all 3 AFPMs is approximately $1 \mathrm{~mm}$ by $1.2 \mathrm{~mm}$ with approximately 2 micron resolution (Figure 2 ). Achieving mechanical precisions sufficient to locate the $O E$ device and the ends of the fibers within this field of view is fairly straightforward. Object-recognition algorithms written by LLNL allow the AFPM to determine the initial locations of the $\mathrm{OE}$ device and the fiber; this image analysis takes between 1 second to 6 seconds, depending upon the complexity of the image. The 2-micron resolution of the vision system allows the fiber to be moved to within a few microns of the desired initial position. This is sufficiently accurate to ensure that some coupling of light between the OE device and the fiber will occur. At this point, the AFPM switches to active feedback to perform the sub-micron alignment, that is, the AFPM performs a series of peakfinding motions until the coupling efficiency is maximized.

\section{High-precision Stages}

The high-precision stages were designed and built by MIT after extensive discussions with UTP and Ortel regarding the required resolution and range. The required resolution was determined by the mode size of the UTP waveguide device operating at $830 \mathrm{~nm}$ and the corresponding single-mode fiber to be pigtailed. The resolution of the stages needs to be a small fraction of the waveguide dimensions to ensure high coupling efficiencies. For the AFPMs, the chosen resolutions for the stages were 0.1 micron in the lateral dimensions and 0.5 micron in the longitudinal dimension. The range of the stages was determined by the working volume required by the AFPM to access the interior of the 14-pin DIP package of the Ortel photodiode; for this project, the range of the high-precision stages was chosen to be $25 \mathrm{~mm}$ in each of three translation directions. The issue of whether to include fiber roll in the AFPM created considerable discussion. Ultimately, roll was not included for this project once satisfactory mechanical fixturing was designed to accommodate this degree of freedom.

\section{Parts Handling}

One of the original design criteria for the AFPM was that it should operate unattended for at least an hour; this implies that at least 20 pigtails will be performed without operator intervention. The issue of parts handling and feeding becomes important for an automated 
system compatible with a manufacturing environment. Many aspects of the electronics industry are fully automated in terms of feeding parts to the assembly stage, so the technology is well developed. For this project, however, a parts handling scheme was developed which relies upon the operator to load the OE device and the fibers into a tray, the so-called kit tray (Figure 3). A set of six loaded kit trays is placed onto a pallet by the operator who then places the pallet onto the conveyor system of the AFPM. The conveyor system may be chosen to have any length necessary to allow the desired time of unattended operation.

The kit tray is designed for easy placement of the OE device and the fibers with sufficient accuracy to allow alignment by the AFPM. The overall design of the kit tray is standard for all applications. The tray body is cast from hard plastic which allows 25 micron repeatability of the placement of the $O E$ device with respect to the vision system from tray to tray. The kit tray can hold up to four spools of fiber depending upon the type of OE device to be pigtailed. Unfortunately, every OE device has a different geometry so no standardization of the kit tray was possible for the fixture to hold the device. The kit tray body is designed to accommodate a device cartridge which fits onto the top of the kit tray; the device cartridge is one of the few AFPM components which must be custom designed for each OE device application.

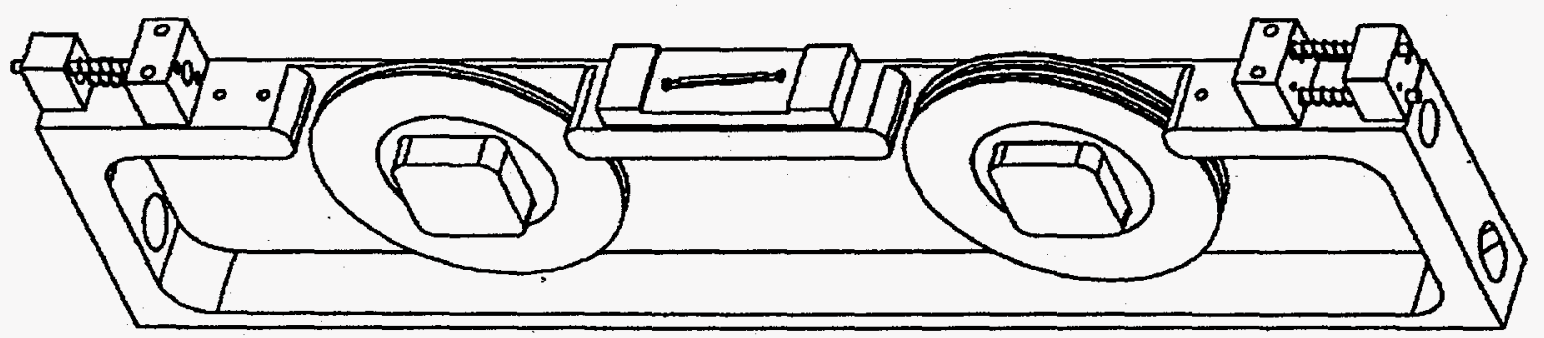

Figure 3 . The kit tray measures $15^{\prime \prime}$ long by $3.5^{\prime \prime}$ high by $1^{\mathrm{n}}$ thick. The tray body is standard for all applications while only the device cartridge in the center is customized for each $O E$ device. The fiber spools can hold at least 2 meters of fiber.

\section{Summary}

Three Automated Fiber Pigtailing Machines (AFPMs) have been designed and built under this ARPA-funded project. The AFPM enables many of the critical technologies to perform automated sub-micron fiber pigtailing compatible with a low-cost manufacturing environment. These technologies include low-cost high-precision stages, computer vision to replace the laborintensive coarse alignment, and many details of parts handling and feeding. Subsequent generations of the AFPM may build upon the design concepts developed here to pigtail fibers to OE devices in more complicated geometries. For example, all applications for this project use epoxy to attach the fibers, so no applications using solder or laser welding have been considered. Also, the stages to manipulate the fibers provide only 3 axes of translation, so no rotational degrees of freedom are available, including the very important roll axis for polarizationdependent applications. The AFPM to reside at LLNL will be used for demonstrations to US industry and for further development of AFPM capabilities.

\section{Acknowledgments}

The author wishes to thank Andy Yang and Anis Husein of the Advanced Research Projects Agency for funding this project. Other team members in this collaboration include Andre Sharon at MIT, Don Bossi at UTP, Ron Moeller at Ortel, and Randy Heyler at Newport/Klinger. At Lawrence Livermore National Laboratory, this work was performed in part under the auspices of the U.S. Department of Energy under contract No. W-7405-Eng-48. 